\title{
Les relations interpersonnelles au sein de la famille recomposée : un état de la littérature
}

\section{Interpersonal relationships within stepfamilies: A review of literature}

\author{
G. Repond*, J. Darwiche \\ Institut de psychologie, faculté des sciences sociales et politiques, université de Lausanne, bâtiment Géopolis, \\ 1015 Lausanne, Suisse
}

Reçu le 4 décembre 2015 ; accepté le 20 juin 2016

\section{Résumé}

Les familles recomposées représentent l'une des configurations familiales actuelles. Cet article propose une revue des études ayant investigué la qualité des relations interpersonnelles au sein de la famille recomposée : la relation entre l'enfant et son beau-parent, entre l'enfant et son parent, la relation conjugale et la relation coparentale au sein du couple recomposé et, plus globalement, le fonctionnement familial. Les résultats disponibles quant à l'impact de ces différentes relations sur les difficultés internalisées et externalisées de l'enfant seront discutés, de même que ceux démontrant les influences réciproques entre certaines de ces relations. Enfin, nous proposons, sur la base de cette revue empirique, quelques pistes de travail susceptibles d'apporter aux professionnels en lien avec ces familles une lecture des enjeux relationnels propres à ces familles.

(C) 2016 Société Française de Psychologie. Publié par Elsevier Masson SAS. Tous droits réservés.

Mots clés : Famille recomposée ; Beaux-parents ; Relations interpersonnelles ; Coparentalité

\section{Abstract}

Stepfamilies are common nowadays. This article reviews studies on the quality of the various interpersonal relationships in stepfamilies: the stepparent-stepchild relationship, the parent-child relationship, the marital and coparenting relationships, and overall family functioning. We discuss the studies' findings on how these relationships are linked and how they affect children's internalized and externalized difficulties. Finally, on

\footnotetext{
* Auteur correspondant.

Adresse e-mail : Gloria.repond@unil.ch (G. Repond). 
the basis of this empirical review, we propose some directions for future work for professionals working with stepfamilies that could improve understanding of the relationship issues specific to stepfamilies. (C) 2016 Société Française de Psychologie. Published by Elsevier Masson SAS. All rights reserved.

Keywords: Stepfamilies; Stepparents; Interpersonal relationships; Coparenting

L'augmentation du taux de divorce ces dix dernières années en Europe a multiplié et redéfini les formes familiales faisant de la famille recomposée une réalité très présente dans notre société actuelle (Damon, 2012). La multiplicité des liens entre les différents membres de la famille recomposée (tels que la relation entre le parent-enfant, les beaux-parents-enfant, relation conjugale et coparentale) fait de l'étude de cette nouvelle forme familiale, une étude complexe mais également très riche.

\section{Systèmes et sous-systèmes des familles recomposées}

Une famille peut être représentée comme un système, composé de plusieurs sous-systèmes relationnels qui s'influencent les uns les autres (Bateson, 1972 ; Minuchin, 1974). Chaque sous-système a ses caractéristiques propres et le fonctionnement de l'un des sous-systèmes ne rend pas forcément compte du fonctionnement des autres sous-systèmes ni du fonctionnement global de la famille. Ainsi, des tensions au sein du sous-système conjugal, c'est-à-dire de la relation affective et sexuelle entre les partenaires, n'impliquent pas obligatoirement la présence de difficultés au niveau du sous-système de la fratrie. Cependant, des influences sont possibles, comme lorsque les tensions débordent du sous-système conjugal pour se répercuter sur les sous-systèmes parents-enfants, altérant la relation que chaque parent entretient avec son enfant.

La famille recomposée se caractérise par un nombre plus important de sous-systèmes relationnels, que la famille traditionnelle : nous pouvons citer notamment le sous-système beau-parent et enfant - qui concerne le lien entre le beau-parent et l'enfant de son conjoint ; le sous-système parent non gardien et enfant - qui se réfère au lien entre le parent biologique n'ayant pas le droit de garde et son enfant; le sous-système de la fratrie recomposée - qui concerne le lien entre les enfants de chaque partenaire, voire celui entre les demi-frères et les demi-sœurs (Anderson, 1999 ; Baham, Weimer, Braver, \& Fabricius, 2008) ; ou encore le sous-système des ex-conjoints, qui touche à la relation entre les parents séparés ou divorcés (Browning \& Artelt, 2012 ; Fine \& Kurdek, 1994 ; Maccoby \& Mnookin, 1992 ; Parent et al., 2008 ; Widmer, Favez, Aeby, De Carlo, \& Doan, 2012). Le fonctionnement d'une famille recomposée est, par ailleurs, influencé par de multiples aspects, tels que les questions de droit et de reconnaissance légale du beau-parent, ainsi que l'attribution des pensions alimentaires, du droit de garde et de l'autorité parentale (Bray \& Berger, 1990 ; Hetherington \& Stanley-Hagan, 1999 ; Sauer \& Fine, 1988 ; White \& Gilbreth, 2001).

La prise en compte de l'entièreté des dynamiques relationnelles ainsi que des variables juridiques et sociales qui influencent le fonctionnement d'une famille recomposée dépasse le cadre de cette revue ; nous nous centrons ici sur les études qui se sont spécifiquement intéressées à évaluer les relations intrafamiliales de la famille recomposée. Nous prenons ainsi en compte les résultats de recherche qui concernent : 
- la relation entre le beau-parent et l'enfant (sous-système beau-parent et enfant) ;

- la relation entre le parent et l'enfant (sous-système parent-enfant) ;

- la relation au sein du couple conjugal (sous-système conjugal) ;

- la relation au sein du couple coparental (sous-système coparental) ;

- la relation entre tous les membres de la famille (système familial).

Les recherches portant sur les familles recomposées se sont développées à partir des années 1980, en particulier aux États-Unis (Clingempeel, Brand, \& Ievoli, 1984 ; Mills, 1984) ; elles sont encore peu présentes en Europe. L'effort des chercheurs s'est porté jusqu'ici sur les caractéristiques de la relation entre le beau-parent et l'enfant, probablement car cette relation se construit suite à la recomposition et qu'elle est susceptible d'influencer le fonctionnement familial (Bray, 1988). A contrario, la relation entre le parent gardien et son enfant n'a reçu que peu d'attention. De même, la relation coparentale entre le parent biologique et son nouveau conjoint n'a que rarement été évaluée dans les études empiriques alors qu'elle représente un aspect important du fonctionnement d'une famille recomposée.

Cette revue de littérature rapporte les principaux résultats d'études ayant investigué la qualité des relations au sein de la famille recomposée, leurs influences mutuelles et leur impact sur l'enfant.

\section{La relation entre le beau-parent et l'enfant}

L'absence de normes sociales quant au rôle de beau-père ou de belle-mère peut compliquer la tâche du beau-parent qui souhaite prendre une place vis-à-vis de l'enfant et développer une relation de qualité avec ce dernier. Le beau-parent peut en effet ne pas comprendre dans un premier temps s'il est attendu de lui d'établir une relation amicale et/ou parentale avec l'enfant (Cherlin, 1978 ; Damon, 2012 ; Gosselin \& David, 2005). Pourtant, les données montrent qu'une relation de qualité entre le beau-parent et l'enfant est essentielle au «bon équilibre » de la famille recomposée (Widmer et al., 2012), mais que celle-ci prend du temps à se stabiliser (Bray \& Berger, 1993).

\subsection{La relation entre le beau-père et l'enfant}

Une relation harmonieuse entre le beau-père et l'enfant semble être associée à un niveau bas de difficultés chez l'enfant. L'étude de White et Gilbreth (2001), menée sur un échantillon de 189 familles, indique une forte association entre la qualité de la relation entre le beau-père et l'enfant (sur la base de questionnaires autoreporté de l'enfant) et un faible niveau de difficultés internalisées (tristesse ou sentiment d'infériorité) et externalisées (impulsivité, agitation) chez l'enfant (questionnaires rempli cette fois-ci par la mère de l'enfant). La formation d'une relation de qualité apparaît dépendre du rôle que le beau-père prend auprès de l'enfant : elle est meilleure lorsque le beau-père ne tente pas d'imposer sa propre autorité et qu'il joue un rôle de soutien de l'autorité parentale (Bray, 1988 ; Walsh, 1991) ; elle est problématique lorsque le beau-père est contrôlant ou manifeste un désengagement vis-à-vis de l'enfant. Dans ce dernier cas, on observe davantage de difficultés de comportement chez l'enfant ainsi que des attitudes de rejet vis-à-vis du beau-père (Hetherington, 1987).

La représentation que le beau-père se fait de son rôle est également déterminante, et ce, indépendamment de l'âge de l'enfant ou du temps de vie commune (Marsiglio, 1992). Par exemple, plus le beau-père s'autorise à jouer un rôle parental, plus il perçoit sa relation à l'enfant comme étant de qualité (Walsh, 1991). 
L'établissement de cette relation reste cependant délicat, quelle que soit l'attitude du beau-père ou la représentation qu'il se fait de son rôle. Ses tentatives pour créer une relation chaleureuse et proche, par exemple à travers des activités communes, peut aussi occasionner des réticences chez l'enfant qui peut percevoir le nouveau compagnon comme un rival pour l'amour de la mère (Browning \& Artelt, 2012 ; Hetherington \& Jodl, 1994).

\subsection{La relation entre la belle-mère et l'enfant}

Le rôle de belle-mère se distingue quelque peu de celui de beau-père puisque bien qu'il n'existe pas de normes sociales quant au rôle d'un beau-parent, certaines représentations ou stéréotypes suggèrent qu'une belle-mère devrait s'engager davantage comme « parent » qu'un beau-père, tant dans l'implication relationnelle avec l'enfant que dans la gestion du foyer (Cherlin, 1978 ; Damon, 2012). Il serait ainsi attendu d'une belle-mère qu'elle joue un rôle de « remplaçante » de la mère, qu'elle s'investisse de manière importante envers l'enfant et prenne la responsabilité du bien-être de la nouvelle famille (Cadolle, 2013 ; Orchard \& Solberg, 1999 ; Walsh, 1991). Plusieurs études montrent que les belles-mères vivent des niveaux de stress et de culpabilité plus importants que les beaux-pères, du fait de l'ambiguïté associée à leur rôle, qui est celui d'un « beau-parent responsable de l'harmonie de la famille » (voir la méta-analyse de Gosselin \& David, 2005 ; Nielsen, 1999).

Toutefois, nous pouvons aussi considérer que lorsque la belle-mère ne prend pas le rôle de « remplaçante » de la mère, mais soutient le père dans son rôle de parent et développe une relation amicale avec les enfants, ces derniers réussissent à développer une relation positive avec elle (Théry \& Dhavernas, 1993). Cette attitude amicale et parentale à la fois, complémentaire de celui de la mère biologique, pourrait aider les enfants à ne pas se sentir pris dans un conflit de loyauté entre leur mère et leur belle-mère (Orchard \& Solberg, 1999). L'étude de King (2007) avec un échantillon de 294 jeunes adolescents (via des questionnaires autoreportés) vivant avec leurs pères et leurs belles-mères confirme cette hypothèse. En effet, ces adolescents se percevaient aussi proches de leur mère que de leur belle-mère. Cette configuration de recomposition apparaît ainsi ne pas empêcher les adolescents de maintenir un lien de qualité avec leur mère tout en développant une relation proche avec leur belle-mère.

En conclusion, nous pouvons souligner que la qualité de la relation entre l'enfant et son beauparent semble être un facteur important pour le bien-être de l'enfant, quel que soit le genre du beauparent. L'attitude du beau-parent, amicale, non-contrôlante et soutenante du parent biologique permettrait ainsi à l'enfant de mieux s'adapter au contexte de la recomposition.

\section{La relation entre le parent et son enfant}

Les recherches portant sur les relations interpersonnelles au sein des familles recomposées ont peu investigué la relation parent-enfant, partant du principe qu'il s'agissait là d'une relation bien établie et stable ne souffrant pas de la recomposition (Cartwright, 2008 ; Mills, 1984 ; Visher \& Visher, 1993). En réalité, le parent biologique se retrouve face au défi de maintenir une relation harmonieuse avec son enfant, tout en investissant la relation conjugale naissante et en essayant de favoriser le lien entre son nouveau conjoint et l'enfant (Smith, 2008). La relation parent-enfant peut alors être malmenée par le stress engendré par la recomposition, ce qui a en effet été observé, en particulier lors des deux premières années de vie commune (Bray \& Berger, 1993 ; Cartwright, 2008 ; Hetherington \& Clingempeel, 1992 ; Hetherington \& Stanley-Hagan, 1999). 


\subsection{La relation entre le père et son enfant}

Les résultats concernent plus souvent le père en situation de parent non gardien, la garde de l'enfant étant généralement attribuée à la mère (OFS, 2008). Les quelques données disponibles à propos de la relation entre le père gardien et son enfant montrent que plus l'enfant se sent dans une relation de proximité avec son père et moins il présente de difficultés internalisées (symptômes dépressifs et détresse psychique) et de difficultés externalisées (comportements délinquants) (King, 2007). Par ailleurs, la qualité de la relation entre le père gardien et son enfant en situation de famille recomposée n'apparaît pas différer de la qualité de la relation entre le père et l'enfant en famille intacte (Sauer \& Fine, 1988). C'est donc surtout la qualité de la relation qui impacte l'enfant que la structure de la famille dans laquelle il grandit (qu'elle soit traditionnelle, recomposée avec un père gardien ou recomposée avec un père non gardien).

Dans le cas des pères non gardiens de l'enfant, deux résultats apparaissent importants. Le premier démontre une tendance des pères non gardiens à se distancer de leur enfant (Sauer \& Fine, 1988), voire à rester en retrait pour ne pas obstruer la nouvelle relation entre l'enfant et son beau-père (Bray \& Easling, 2005) ; le second indique que lorsque le père non gardien réussit à maintenir un lien à son enfant, celui-ci présente moins de difficultés externalisées. White et Gilbreth (2001) ont ainsi interrogé (via questionnaires autoreportés) 189 mères sur la perception qu'elles avaient de la relation entre leur enfant et le père non gardien. Ces dernières reportent en effet de moins de manifestations de comportements impulsifs et d'hyperactivité.

\subsection{La relation entre la mère et son enfant}

Les résultats concernent majoritairement la situation de mères ayant la garde principale de leur enfant. La recomposition marque souvent un changement dans la relation mère-enfant d'où peuvent émerger des tensions et des conflits relationnels : l'enfant n'est plus seul avec sa mère comme lors de la période de vie monoparentale et se retrouve à la « partager » avec le beaupère (Hetherington \& Stanley-Hagan, 2002). Toutefois, les difficultés éprouvées par l'enfant semblent transitoires puisqu'elles disparaissent avec le temps. L'étude longitudinale de Bray et Berger (1993) basée sur 97 familles recomposées et 98 familles de première union (questionnaires autoreportés), montre en effet que la relation mère-enfant en contexte de recomposition est moins bonne à six mois de vie commune, en comparaison à la même relation en famille de première union mais que cette différence disparaît après deux ans et demi de recomposition. Ce résultat se confirme avec une autre étude basée sur les réponses autoreportées de 111 familles recomposées, qui ne montre aucune différence significative de qualité de la relation mère - enfant en famille recomposée ou nucléaire lorsque les mesures sont prises auprès de familles recomposées établies depuis plusieurs années (Smith, Robertson, Dixon, Quigley, et Whitehead, 2001 in Robertson, 2008).

Les données sur la relation mère-enfant dans la situation où la mère n'est pas le parent gardien sont relativement rares. Parmi les quelques résultats disponibles, Stewart (1999) relève notamment qu'en comparaison aux pères non gardiens, les mères non gardiennes ont des contacts plus réguliers et plus fréquents avec leurs enfants (notamment via téléphone). D'autres études plus récentes mettent en avant l'importance de la fréquence des contacts avec l'enfant d'une part (Pryor, 2008) et la qualité de ces contacts, d'autre part (King, 2007). Plus particulièrement, les enfants qui décrivent se sentir proches de leurs mères non gardiennes (sentiment subjectif d'un lien d'attachement) reportent moins de difficultés internalisées et externalisées (King, 2007). 
Les différents résultats de cette section sur la relation entre le parent et son enfant montrent que la qualité du lien entre le parent et son enfant est primordiale pour le bien-être de l'enfant, en particulier pour les pères, qu'ils soient gardiens ou non, et pour les mères non gardiennes. Pour les mères gardiennes, la relation à l'enfant est surtout affectée au moment de la transition au foyer recomposé ; avec le temps, les difficultés s'atténuent, voire disparaissent.

\section{La relation conjugale au sein du couple recomposé}

La création d'un nouveau foyer familial a un impact sur la qualité de la relation conjugale. L'un des deux partenaires au moins a fait l'expérience d'un échec marital et des conséquences d'une séparation. Les études montrent que les couples recomposés font face à un agenda chargé, puisqu'ils ont la tâche de faire reconnaître leur « nouveau » couple auprès de leurs enfants et de leur entourage familial ainsi que celle de réussir à créer une relation conjugale forte (Bray, 2005). Cet agenda chargé peut expliquer que la satisfaction conjugale des couples recomposés diminue avec le temps et qu'un tiers des couples recomposés se séparent lors des deux premières années de vie commune (Bray, 2005 ; Bray \& Kelly, 1998).

Les données montrent que la détresse conjugale des couples recomposés est renforcée par la présence d'un enfant issu d'une précédente union (Bray \& Berger, 1993 ; Halford, Nicholson, \& Sanders, 2007 ; Marsiglio, 1992 ; Nicholson, Phillips, Whitton, Halford, \& Sanders, 2007 ; White \& Booth, 1985). Menée sur un large échantillon de 455 couples, l'étude de Van Eeden-Moorefield et Pasley (2008) relève une forte association entre la détresse conjugale (évaluée par des questionnaires autoreporté de satisfaction conjugale) et la présence d'un enfant issu de la précédente union de l'un des partenaires. Cette observation est nuancée par les résultats de l'étude de Fine et Kurdek (1995). En effet, sur un échantillon de 111 familles avec belle-mère et 92 familles avec beau-père, les résultats issus des questionnaires montrent que c'est la qualité de la relation entre le beau-parent et l'enfant qui est déterminante pour la satisfaction conjugale, et non simplement la présence d'un enfant. Ainsi, lorsque le beau-père se sent proche affectivement de l'enfant (Orleans, Palisi, \& Caddell, 1989) et à l'aise dans son rôle, c'est-à-dire qu'il réussit à établir une relation de confiance avec l'enfant, qu'il se sent respecté dans son autorité et soutenu par le parent des enfants (Beaudry, Parent, Saint-Jacques, Guay, \& Boisvert, 2001), sa satisfaction conjugale est plus élevée. Ces données n'indiquent pas si le développement d'une relation conjugale solide est un préalable à son engagement vis-à-vis de l'enfant. Certains auteurs avancent, cependant, l'hypothèse que l'espoir du beau-parent de s'engager dans une relation conjugale stable précède l'investissement de la relation à l'enfant (Parent et al., 2008).

L'association entre satisfaction conjugale et qualité du lien avec l'enfant semble se modifier dans le temps, du moins dans les familles recomposées avec beau-père (Bray \& Berger, 1993). Au début de la recomposition, autour de six mois après le remariage, la satisfaction conjugale du beaupère est plus élevée lorsqu'il ne se sent pas obligé d'assumer un rôle parental ou de développer une relation de proximité avec l'enfant. Après deux ans et demi de mariage, la satisfaction conjugale du beau-père est au contraire plus élevée s'il perçoit sa relation à l'enfant comme proche. Finalement, l'association entre la satisfaction conjugale et la qualité de la relation à l'enfant disparaît sept ans après le remariage. Ce résultat suggère que la satisfaction du beau-père quant à sa relation conjugale ne dépend plus autant de son rôle de beau-parent après plusieurs années de vie commune.

Le couple recomposé ne se limite toutefois pas au couple conjugal. Les conjoints forment également une équipe de coparents et la qualité de cette relation va avoir une importance pour le fonctionnement de la famille recomposée. 


\section{La relation coparentale au sein du couple recomposé}

Les travaux sur la relation coparentale au sein du couple recomposé, qui correspond au lien entre le parent et le beau-parent en tant que responsables des questions liées aux enfants, sont pratiquement inexistants. Les recherches sur la coparentalité ont en effet porté jusqu'ici sur la relation coparentale entre parents divorcés (par exemple : Adamsons, Pasley, Fine, \& Harvey, 2006 ; Ahrons, 1981) ou entre parents de première union (Favez \& Frascarolo, 2013 ; Frascarolo, Despland, Tissot, \& Favez, 2012 ; McHale, 1997). L'importance de cette relation au sein des familles traditionnelles ayant été largement démontrée, il nous apparaît utile de traiter cet aspect dans la famille recomposée.

\subsection{Quelques éléments de définition}

La coparentalité est une notion initialement issue des travaux de Minuchin faisant référence au « sous-système exécutif parental » (Minuchin, 1974), c'est-à-dire le couple parental au sein de la famille. Une relation coparentale de qualité peut être définie comme la capacité des adultes responsables de l'enfant à se coordonner, à co-créer une structure protectrice pour l'enfant et à se soutenir mutuellement dans leurs rôles de parents (McHale \& Irace, 2011 ; McHale, Kuersten-Hogan, Lauretti, \& Rasmussen, 2000). La coparentalité implique généralement quatre dimensions : l'intégrité familiale (c'est-à-dire les comportements de chaque parent ou beau-parent visant à promouvoir ensemble l'unité de la famille), les conflits, l'affection et les comportements de dénigrement (McHale, 1997). La coparentalité est présente dans tout type de famille, qu'elle soit par exemple nucléaire, recomposée, adoptive ou homoparentale. Dans les familles traditionnelles, un coparentage harmonieux, caractérisé par une coordination élevée entre les coparents et des relations chaleureuses, est associé à une satisfaction conjugale plus élevée (Fainsilber Katz \& Gottman, 1996 ; Favez \& Frascarolo, 2013 ; Schoppe-Sullivan, Mangelsdorf, Frosch, \& McHale, 2004) et permet une meilleure adaptation socioémotionnelle de l'enfant (Favez, Frascarolo, \& Lavanchy Scaiola, 2011 ; Frascarolo-Moutinot, Suardi, Lavanchy-Scaiola, \& Favez, 2007 ; McHale, 2007).

Dans une famille recomposée, la coparentalité présente certaines spécificités. Soulignons avant tout que la coparentalité de l'enfant en famille recomposée peut inclure jusqu'à quatre coparents (parents biologiques et leurs nouveaux conjoints). Il sera alors question non seulement de l'accord coparental dans chaque sous-système coparental mais également de la coordination entre tous les coparents, que l'on peut qualifier de supracoparentage (Frascarolo et al., 2012). Dans cette revue, nous nous centrons sur la relation de coparentalité entre le parent biologique et le beau-parent.

Un premier aspect concerne l'engagement du beau-parent en tant que coparent : de quelle manière souhaite-t-il s'engager et dans quelle mesure se sent-il autorisé par le parent biologique à intervenir ? En effet, il est tout à fait possible d'imaginer un coparentage fonctionnel où l'un des deux parents est moins engagé, voire désengagé (Bray \& Berger, 1993) ou encore où l'un est particulièrement investi (notamment le parent biologique). Un second aspect touche au nécessaire ajustement entre les coparents : dans quels domaines et dans quelles proportions l'engagement du beau-parent est-il souhaité ? Dans l'idéal, on pourrait imaginer que le beau-parent fonctionne comme membre à part entière de l'équipe parentale même si le parent biologique reste la principale « porte d'accès » à l'enfant, c'est-à-dire le référent de l'enfant (Parent et al., 2008). Dans tous les cas, et même s'il est important de garder à l'esprit qu'une coparentalité de qualité peut prendre une forme différente de celle constatée chez les familles de première union, fonctionner en tant 
qu'équipe coparentale va impliquer pour les partenaires des ajustements importants qui devront être régulièrement rediscutés au cours du temps (Parent et al., 2008).

\subsection{Quelques données de recherche}

À notre connaissance, il n'existe que quatre études portant sur la qualité de la relation coparentale dans le couple recomposé (Braithwaite, McBride, \& Schrodt, 2003 ; Schrodt \& Braithwaite, 2011 ; Orleans, Palisi, \& Cadell (1989); Favez, Widmer, Doan, \& Tissot, 2015).

La première étude (Braithwaite et al., 2003) comprend 22 familles recomposées établies depuis six ans en moyenne. L'analyse d'un « journal de bord » rempli quotidiennement par le parent et le beau-parent pendant deux semaines. Il était demandé aux participants de décrire l'interaction coparentale, d'estimer l'efficacité de cette interaction ou encore d'en évaluer le niveau de conflit. Les résultats montrent ainsi que les interactions coparentales sont peu fréquentes et peu conflictuelles, et que les beaux-parents initient rarement les interactions au sujet de l'enfant avec le partenaire. Les bas niveaux de conflit et de tension observés suggèrent que ces familles ont trouvé un certain équilibre au niveau coparental. La deuxième recherche (Schrodt \& Braithwaite, 2011), cette fois-ci basée sur des questionnaires autoreportés et menée sur un échantillon de 127 couples, montre que les couples recomposés dont la coparentalité se fait sur un mode coopératif et soutenant se disent plus satisfaits maritalement. La qualité de la relation coparentale semble donc être liée à la qualité de la relation conjugale, comme cela a été largement démontré dans les familles de première union (par exemple : Frascarolo-Moutinot et al., 2007 ; Schoppe-Sullivan et al., 2004). Cette hypothèse est également soutenue par l'étude de Orleans et al. (1989) qui retrouve, avec un échantillon de 60 familles recomposées, que plus le beau-père reporte être en accord avec sa conjointe concernant les décisions relatives à l'enfant et plus sa satisfaction conjugale est élevée (questionnaires autoreportés).

La récente étude de Favez et al. (2015) propose une analyse détaillée de la coparentalité avec un échantillon de 300 mères (questionnaires autoreportés), dont la moitié est en famille recomposée et l'autre moitié en famille de première union. Les auteurs explorent trois des dimensions de la coparentalité : l'intégrité familiale, le conflit et le dénigrement. Les résultats montrent tout d'abord que les comportements coparentaux sont plus fréquents entre la mère et le beau-parent qu'entre la mère et son ex-conjoint. Toutefois, la fréquence des comportements coparentaux reste globalement moins élevée en comparaison avec les familles de première union.

Deuxièmement, les auteurs ne trouvent pas de lien entre la satisfaction conjugale de la mère et la qualité du lien coparental avec le beau-père (dimension d'intégrité familiale) alors que ce lien est observé pour les mères des familles de première union. Par contre, et comme pour les mères des familles de première union, plus la mère est satisfaite au niveau conjugal, et moins elle relève de problèmes au niveau coparental avec son partenaire (dimensions de conflit et dénigrement). Il existe donc bien une influence entre relations conjugale et coparentale dans les familles recomposées mais seulement sur certaines dimensions de la coparentalité. Selon les auteurs, cela pourrait s'expliquer par le fait que les sous-systèmes conjugal et coparental sont partiellement séparés dans les familles recomposées.

Ces quelques études soulignent que la coparentalité n'est pas l'apanage des familles traditionnelles puisque des comportements coparentaux sont observés au sein des couples recomposés. Cependant, cette coparentalité apparaît prendre des formes spécifiques : en particulier, il s'agit d'être attentif au conflit coparental et au dénigrement puisque ces dimensions sont associées à la présence de détresse conjugale. 


\section{Le fonctionnement familial}

Reconstruire un nouveau modèle familial dans une société pour qui la famille de première union reste la norme est un défi pour les familles recomposées (Papernow, 1984 ; Walsh, 1991). La création d'une vie de famille apparaît souvent comme un des buts de la famille recomposée. Ce but peut être compliqué par le nombre d'enjeux relationnels à appréhender simultanément, pour les nouveaux partenaires et pour les enfants en présence (Allan, Hawker, \& Crow, 2008). Il n'existe que peu de données empiriques sur le fonctionnement global de la famille recomposée en tant que tel, c'est-à-dire sur la perception par les membres de la famille de la qualité du climat familial (Bandura, 1993 ; Jones \& Prinz, 2005) ou sur l'observation des interactions entre les membres de la famille réunis.

Les quelques données disponibles portent sur le sentiment de cohésion familiale, c'est-à-dire le sentiment pour les membres de la famille de se percevoir comme une entité familiale. Une première étude comparant 28 familles de première union et 28 familles recomposées rapporte que le sentiment de cohésion est moins élevé chez les familles recomposées en comparaison avec les familles de première union (sur la base de questionnaires autoreportés); de plus, les membres des familles recomposées perçoivent leur fonctionnement comme moins flexible, c'est-à-dire qu'il est plus difficile de trouver un équilibre familial, entre stabilité et changements (Pink \& Wampler, 1985). D'autres études observent également que les familles recomposées depuis six mois environ reportent moins de cohésion, moins de communication et une responsivité affective moins élevée (c'est-à-dire la capacité de chaque membre à exprimer des affects de manière appropriée) que les familles de première union (Bray, 1988 ; Bray \& Berger, 1993). Toutefois, ces différences ne s'observent plus après deux ans et demi de vie commune, indiquant que le sentiment de cohésion apparaît se développer avec le temps.

\section{Discussion}

Cette revue de la littérature synthétise les résultats d'études ayant évalué les relations interpersonnelles au sein des familles recomposées : la relation entre le beau-parent et l'enfant, la relation entre le parent et son enfant, la relation conjugale, la relation coparentale au sein du couple recomposé et la dynamique familiale. Si certains de ces niveaux, comme la relation beau-parent et enfant et la relation conjugale, ont été largement étudiés, d'autres, comme la relation coparentale, n'ont à ce jour que peu été explorés.

\subsection{Les principales influences}

Les résultats disponibles présentent la relation entre le beau-parent et l'enfant comme un facteur clé pour l'ajustement de la famille puisque, comme cela a été démontré, plus cette relation est de qualité et moins on observe de difficultés internalisées et externalisées chez l'enfant. Cette relation prend cependant du temps à se mettre en place et à se stabiliser : il est essentiel que le beau-parent se sente « légitimé » à entrer en relation avec l'enfant de son partenaire, à prendre une place au sein du foyer recomposé tout en veillant à ne pas interférer dans les autres relations, notamment celle entre l'enfant et son parent biologique non gardien. Néanmoins, quant tout se passe bien, les études montrent que cela peut avoir des effets généraux positifs sur la constellation relationnelle de l'enfant : un lien de qualité à son beau-parent est associé à un lien de qualité à son parent et à son parent non gardien. Ces données suggèrent que le risque d'un conflit de 
(ajouter une référence ici) envers l'un ou l'autre de ses parents est diminué pour l'enfant lorsqu'il entretient des liens de qualité avec les différents adultes qui ont sa charge.

Les résultats des études présentées relèvent également des influences mutuelles entre la satisfaction au sein du couple conjugal et la qualité de la relation entre le beau-parent et l'enfant. Plus précisément, un beau-parent engagé dans une relation conjugale satisfaisante et durable aura également une meilleure perception de sa relation à l'enfant. Cette association peut être interprétée de deux manières. Premièrement, du fait de l'absence de lien biologique entre le beau-parent et l'enfant, la relation entre eux étant subordonnée à la relation conjugale : si le couple conjugal se défait, la relation à l'enfant également, dans la grande majorité des cas. Ensuite, contrairement aux familles de première union, les relations beau-parentale et conjugale se créent simultanément. De ce fait, nous pouvons considérer ces relations comme « deux vases communicants », dont les frontières sont perméables. Cela permet de comprendre que les difficultés survenant dans l'une de ces relations, par exemple au sein du couple conjugal, puissent se disséminer dans l'autre relation, à savoir celle du beau-parent avec l'enfant.

\subsection{La relation coparentale}

Le faible nombre d'études sur la relation coparentale dans les familles recomposées ne nous permet pas d'établir de conclusion sur l'importance de la qualité du lien coparental au sein de la famille recomposée. Il est frappant de constater que même les ouvrages dédiés à la coparentalité (par exemple : McHale \& Lindahl, 2011) ne mentionnent à aucun moment la famille recomposée. Les données à disposition indiquent pourtant que les comportements coparentaux sont bien présents (même s'il peut s'agir d'un coparentage non-soutenant ou déséquilibré) entre le parent biologique et le beau-parent de l'enfant malgré une structure familiale différente. De plus, amples recherches sur la coparentalité dans les familles recomposées permettront de mettre en avant les spécificités propres à cette structure familiale et, le cas échéant, de modifier les représentations sociales du couple recomposé. En effet, il est courant aujourd'hui de considérer les liens que chaque membre du couple développe avec l'enfant - celui du parent biologique et de son enfant et celui du beau-parent avec l'enfant - mais il est moins commun de considérer les partenaires comme formant une équipe coparentale et travaillant de concert pour le bien de l'enfant.

\section{Quelques pistes de travail avec les familles recomposées}

Les résultats présentés ci-dessus dévoilent une partie de la complexité des relations interpersonnelles dans les familles recomposées. Ce que les recherches nous ont appris sur le fonctionnement d'une famille traditionnelle ne peut être utilisé comme seule carte de compréhension de la famille recomposée : cela reviendrait en effet à « tenter de se repérer dans les rue de New York avec une carte de la ville de Boston $»$ (Papernow, 2008, p. 432). Nous proposons ici quelques considérations pour le travail avec les familles recomposées, sur la base des grandes lignes dessinées par les résultats de cette revue (Ganong \& Coleman, 2004).

\subsection{Complexité de la structure familiale}

La configuration de la famille recomposée est variable et complexe, comme nous pouvons l'observer non seulement du fait du nombre des relations à mettre en place et à consolider, du nombre de membres de la famille présents ou absents selon les jours de la semaine (répartition de la garde des enfants), mais aussi de part les influences réciproques de ces différentes relations. Il 
s'agit alors de reconnaître, sans les exagérer, les difficultés que peut traverser la famille recomposée et le temps nécessaire dont elle aura besoin pour que ces différentes relations s'établissent et pour que les frontières entre les différents sous-systèmes se créent.

\subsection{Relation entre l'enfant et son beau-parent}

La qualité de la relation entre l'enfant et son beau-parent est un élément clé pour le bon fonctionnement de la famille recomposée. Le désengagement ou au contraire le désir du beauparent de se rapprocher ou d'endosser un rôle parental de manière précipitée peuvent mettre à mal la relation à l'enfant.

Le fait que la présence ou l'absence de ces difficultés chez l'enfant soit de manière répétée associée dans les études à la qualité de la relation avec le beau-parent souligne l'importance du lien de l'enfant à son beau-parent et la nécessité de soutenir le beau-parent dans ses tâches.

\subsection{Relation au parent non gardien}

La relation de l'enfant à son parent non gardien, que celui-ci soit présent ou non dans la vie de l'enfant, impacte sur la relation au beau-parent. Quelle que soit l'attitude du beau-parent, il apparaît ici important de soutenir l'enfant lui-même, surtout lorsqu'il est tenté d'idéaliser son parent non gardien. Il s'agira en effet de l'aider à donner une place à chacune de ces relations et à sortir d'un éventuel conflit de loyauté (Gosselin \& David, 2005 ; Saint-Jacques, 1990). En effet, la loyauté que l'enfant peut ressentir envers le parent non gardien peut, en effet, entraîner le rejet du beau-parent, parfois vu comme la « cause » du divorce parental.

\subsection{Conflit de loyauté de l'enfant}

Le conflit de loyauté dans lequel peut être pris l'enfant n'est pas l'apanage des familles recomposées. Néanmoins, la séparation des parents biologiques peut mettre l'enfant en situation de « porte-à-faux », dans l'impossibilité de satisfaire chaque parent (Buchanan, Maccoby, \& Dornbush, 1991). L'arrivée du beau-parent dans la nouvelle constellation familiale peut encore accroître ce sentiment de conflit de loyauté. Toutefois, il est essentiel de garder à l'esprit que la résolution (ou non) de ce conflit passe en premier lieu par les actions des adultes environnant l'enfant et non pas par l'enfant lui-même (Coleman, Fine, Ganong, Downs, \& Paux, 2001). Certaines interventions (c'est juste ?), comme le projet AIDES (Lessard, Chamberland, \& Léveillé, 2007), promeuvent le travail avec les parents pour les soutenir dans leurs pratiques parentales mais prennent aussi en compte les besoins spécifiques de l'enfant (renforcer l'estime de soi, soutien aux devoirs, etc.). Dans un contexte de conflit de loyauté, la collaboration entre tous les parents (biologiques et beaux-parents) via un supracoparentage peut permettre d' aider l'enfant à sortir de cette position délicate pour lui et cause de grande souffrance.

\subsection{Ancienneté du lien parent-enfant par rapport au lien conjugal}

Au-delà du fait que la relation qui unit le parent à son enfant et la relation conjugale soit intrinsèquement différentes, le lien unissant le parent biologique à l'enfant précède celui qui unit le nouveau couple. Le parent et son enfant partagent une histoire et des souvenirs communs, des liens forts et solides alors que le couple conjugal qui en est encore à se découvrir, est plus «fragile ». Il s'agit ici de soutenir le parent lui-même et d'aider le beau-parent à soutenir le parent, 
dans une triple tâche : celle de préserver la relation déjà existante à l'enfant, de s'engager dans une relation conjugale et de favoriser le lien entre l'enfant et le beau-parent (Smith, 2008).

\subsection{Coparentalité dans les familles recomposées}

Ce que nous savons du lien coparental au sein des familles traditionnelles ou entre ex-conjoints ne peut être repris tel quel pour comprendre les spécificités du fonctionnement comme coparents entre un parent biologique et son conjoint. Le fait de s'engager de manière similaire auprès de l'enfant, par exemple, peut ne pas être adéquat dans les premiers temps de la recomposition, ni même à plus long terme. Les professionnels peuvent ici soutenir le couple dans cet art de la coparentalité, en les aidant à trouver les ajustements qui leur permettront de travailler en équipe de manière coordonnée.

\subsection{Pertes et changements}

La famille recomposée se crée suite à de nombreux changements et pertes : séparation du couple, perte totale ou en partie du contact avec le parent non gardien, adaptation à une période de vie monoparentale, changement de lieu de vie et de lieu de scolarité pour l'enfant, etc. À cela s'ajoute également la perte de certains repères, projets et relations (D'Amore, Gresse, \& Pauss, 2011 ; Goldbeter-Merinfeld, 2010). Ces changements peuvent néanmoins aussi représenter une source de gain, tant pour le parent qui se trouve à partager les difficultés du quotidien avec son partenaire que pour l'enfant qui peut trouver chez son beau-parent un nouvel adulte de référence. Il s'agit donc pour le professionnel de garder en tête le contexte d'émergence de la famille recomposée (pertes et changements) mais également de valoriser les ressources de la famille.

\section{Conclusion}

La prise en compte des relations interpersonnelles au sein de la famille recomposée permet d'avancer dans la compréhension de ce que ses membres peuvent vivre comme expérience d' «être une famille ». De futures recherches nous semblent néanmoins indispensables pour augmenter les connaissances sur le fonctionnement des familles recomposées, notamment en ce qui concerne le développement d'une relation coparentale et d'un sentiment de cohésion familiale.

\section{Déclaration de liens d'intérêts}

Les auteurs déclarent ne pas avoir de liens d'intérêts.

\section{Références}

Adamsons, K., Pasley, K., Fine, M. A., \& Harvey, J. H. (2006). Coparenting following divorce and relationship dissolution. In M. A. Fine, \& J. H. Harvey (Eds.), Handbook of divorce and relationship dissolution (pp. 241-261). Mahwah, NJ: Lawrence Erlbaum Associates.

Ahrons, C. R. (1981). The continuing coparental relationship between divorced spouses. American Journal of Orthopsychiatry, 51, 415-428.

Allan, G., Hawker, S., \& Crow, G. (2008). Kinship in stepfamilies. In J. Pryor (Ed.), The international handbook of stepfamilies: Policy and practice in legal, research, and clinical environments (pp. 322-344). New Jersey: John Wiley \& Sons. 
Anderson, E. R. (1999). Chapter V. Sibling, half sibling, and stepsibling relationships in remarried families. Monographs of the Society for Research in Child Development, 64, 101-126.

Baham, M. E., Weimer, A. A., Braver, S. L., \& Fabricius, W. V. (2008). Sibling relationships in blended families. In J. Pryor (Ed.), The international handbook of stepfamilies: Policy and practice in legal, research, and clinical environments (pp. 175-207). New Jersey: John Wiley \& Sons.

Bandura, A. (1993). Perceived self-efficacy in cognitive development and functioning. Educational Psychologist, 28 , $117-148$.

Bateson, G. (1972). Steps to an ecology of mind: Collected essays in anthropology, psychiatry, evolution, and epistemology. University of Chicago Press.

Beaudry, M., Parent, C., Saint-Jacques, M. C., Guay, S., \& Boisvert, J. M. (2001). Validation of a questionnaire to assess the difficulties of couples in stepfamilies. Journal of Divorce \& Remarriage, 35, 155-172.

Braithwaite, D. O., McBride, M. C., \& Schrodt, P. (2003). "Parent teams" and the everyday interactions of coparenting in stepfamilies. Communication Reports, 16, 93-111.

Bray, J. H. (1988). Children's development during early remarriage. In E. M. Hetherington, \& J. D. Arasteh (Eds.), Impact of divorce, single parenting, and stepparenting on children (pp. 279-298). Hillsdale: Lawrence Erlbaum Associates.

Bray, J. H. (2005). Family therapy with stepfamilies. In J. L. Lebow (Ed.), Handbook of clinical family therapy (pp. 497-515). Hoboken, NJ: Wiley.

Bray, J. H., \& Berger, S. H. (1990). Noncustodial father and paternal grandparent relationships in stepfamilies. Family Relations, 39, 414-419.

Bray, J. H., \& Berger, S. H. (1993). Developmental issues in stepfamilies research project: Family relationships and parent-child interactions. Journal of Family Psychology, 7, 76-90.

Bray, J. H., \& Easling, I. (2005). Remarriage and stepfamilies. In W. M. Pinsof, \& J. L. Lebow (Eds.), Family psychology: The art of the science (pp. 267-294). New York: Oxford.

Bray, J. H., \& Kelly, J. (1998). Stepfamilies: Love, marriage and parenting in the first decade. New York: Broadway Books.

Browning, S., \& Artelt, E. (2012). Stepfamily therapy: A 10-step clinical approach. Washington, DC: American Psychological Association.

Buchanan, C. M., Maccoby, E. E., \& Dornbusch, S. M. (1991). Caught between parents: Adolescents' experience in divorced homes. Child Development, 62, 1008-1029.

Cadolle, S. (2013). Les belles-mères, entre idéal de coparentalité et asymétrie homme/femme. Dialogue, 3, 35-46.

Cartwright, C. (2008). Resident parent-child relationships in stepfamilies. In J. Pryor (Ed.), The international handbook of stepfamilies: Policy and practice in legal, research, and clinical environments (pp. 208-230). New Jersey: John Wiley \& Sons.

Cherlin, A. (1978). Remarriage as an incomplete institution. American Journal of Sociology, 84, 634-650.

Clingempeel, W. G., Brand, E., \& Ievoli, R. (1984). Stepparent-stepchild relationships in stepmother and stepfather families: A multimethod study. Family Relations, 33, 465-473.

Coleman, M., Fine, M. A., Ganong, L. H., Downs, K. J., \& Pauk, N. (2001). When you're not the Brady Bunch: Identifying perceived conflicts and resolution strategies in stepfamilies. Personal Relationships, 8, 55-73.

D’Amore, S., Gresse, K., \& Pauss, V. (2011). Pertes et ressources dans la construction des nouvelles familles : le cas des familles recomposées. Thérapie Familiale, 32, 111-128.

Damon, J. (2012). Les familles recomposées. Paris: PUF.

Fainsilber Katz, L., \& Gottman, J. M. (1996). Spillover effects of marital conflict: In search of parenting and coparenting mechanisms. New Directions for Child and Adolescent Development, 1996, 57-76.

Favez, N., \& Frascarolo, F. (2013). Le coparentage : composants, implications et thérapie. Devenir, 25, 73-92.

Favez, N., Frascarolo, F., \& Lavanchy Scaiola, C. (2011). Stabilité de l'alliance familiale et développement sociocognitif de l'enfant à 5 ans. In C. Z. Gaudron (Ed.), Précarités et éducation familiale (pp. 114-121). Toulouse: Erès.

Favez, N., Widmer, E. D., Doan, M. T., \& Tissot, H. (2015). Coparenting in stepfamilies: Maternal promotion of family cohesiveness with partner and with father. Journal of Child and Family Studies, 24, 1-11.

Fine, M. A., \& Kurdek, L. A. (1994). Parenting cognitions in stepfamilies: Differences between parents and stepparents and relations to parenting satisfaction. Journal of Social and Personal Relationships, 11, 95-112.

Fine, M. A., \& Kurdek, L. A. (1995). Relation between marital quality and (step) parent-child relationship quality for parents and stepparents in stepfamilies. Journal of Family Psychology, 9, 216-223.

Frascarolo, F., Despland, J. N., Tissot, H., \& Favez, N. (2012). Le coparentage, un concept clé pour évaluer le fonctionnement familial. Psychothérapies, 32, 15-22. 
Frascarolo-Moutinot, F., Suardi, F., Lavanchy-Scaiola, C., \& Favez, N. (2007). Articulation entre alliance familiale, coparentage et conjugalité. In G. Bergonnier-Dupuy, \& M. Robin (Eds.), Couples, famille et métamorphoses (pp. 103-127). Toulouse: Erès.

Ganong, L. H., \& Coleman, M. (2004). Stepfamily relationships: Development, dynamics and interventions. New York: Springer Science \& Business Media.

Goldbeter-Merinfeld, E. (2010). Familles recomposées, familles décomposées : banalisation des deuils. In S. D’Amore (Ed.), Nouvelles familles : Approches cliniques et de recherche (pp. 203-222). Bruxelles: De Boeck.

Gosselin, J., \& David, H. (2005). Défis et contraintes des recherches sur les familles recomposées : l'exemple de la relation belle-mère-belle-fille. Psychologie française, 50, 241-254.

Halford, K., Nicholson, J., \& Sanders, M. (2007). Couple communication in stepfamilies. Family Process, 46, 471-483.

Hetherington, E. M. (1987). Family relations six years after divorce. In K. Pasley, \& M. Ihinger-Tallman (Eds.), Remarriage and stepparenting: Current research and theory (pp. 311-329). New York: Guilford Press.

Hetherington, E. M., \& Clingempeel, W. G. (1992). Coping with marital transitions: A family systems perspective. Monographs of the Society for Research in Child Development, 57, 1-238.

Hetherington, E. M., \& Jodl, K. M. (1994). Stepfamilies as settings for child development. In A. Booth, \& J. Dunn (Eds.), Stepfamilies: Who benefits? Who does not (pp. 55-79). New York: Routledge.

Hetherington, E. M., \& Stanley-Hagan, M. (1999). The adjustment of children with divorced parents: A risk and resiliency perspective. Journal of Child Psychology and Psychiatry, 40, 129-140.

Hetherington, E. M., \& Stanley-Hagan, M. (2002). Parenting in divorced and remarried families. In M. Bornstein (Ed.), Handbook of parenting: Being and becoming a parent (3) (2nd ed., 3, pp. 287-315). Mahwah, NJ: Lawrence Erlbaum Associates.

Jones, T. L., \& Prinz, R. J. (2005). Potential roles of parental self-efficacy in parent and child adjustment: A review. Clinical Psychology Review, 25, 341-363.

King, V. (2007). When children have two mothers: Relationships with nonresident mothers, stepmothers, and fathers. Journal of Marriage and Family, 69, 1178-1193.

Lessard, D., Chamberland, C., \& Léveillé, S. (2007). L'évaluation de l'initiative AIDES : pour changer la culture d'aide à l'enfance et à la famille dans des communautés québécoises. Les enfants du Canada. pp. 38-48. Centre d'excellence pour la protection et le bien-être des enfants.

Maccoby, E. E., \& Mnookin, R. H. (1992). Dividing the child: Social and legal dilemmas of custody. Harvard University Press: Cambridge.

Marsiglio, W. (1992). Stepfathers with minor children living at home parenting perceptions and relationship quality. Journal of Family Issues, 13, 195-214.

McHale, J. P. (1997). Overt and covert coparenting processes in the family. Family Process, 36, 183-201.

McHale, J. P. (2007). Charting the bumpy road of coparenthood: Understanding the challenges of family life. Washington, DC: Zero to three.

McHale, J. P., \& Irace, K. (2011). Coparenting in diverse family systems. In American Psychological Association (Ed.), Coparenting: A conceptual and clinical examination of family systems (pp. 15-37). Washington, DC: American Psychological Association.

McHale, J. P., Kuersten-Hogan, R., Lauretti, A., \& Rasmussen, J. L. (2000). Parental reports of coparenting and observed coparenting behavior during the toddler period. Journal of Family Psychology, 14, 220-236.

McHale, J.P., \& Lindahl, K. M. (2011). Coparenting: A conceptual and clinical examination offamily systems. Washington, DC: American Psychological Association.

Mills, D. M. (1984). A model for stepfamily development. Family Relations, 33, 365-372.

Minuchin, S. (1974). Families and family therapy. Cambridge: Harvard University Press.

Nicholson, J., Phillips, M., Whitton, S. W., Halford, W., \& Sanders, M. R. (2007). Promoting healthy stepfamilies: Couples' reasons for seeking help and perceived benefits from intervention. Family Matters, 77, 48-57.

Nielsen, L. (1999). Stepmothers: Why so much stress? A review of the research. Journal of Divorce \& Remarriage, 30 , $115-148$.

Office fédérale de la statistique. (2008). Les familles en suisse, rapport statistique 2008. (Repéré à http://www.bfs.admin.ch/bfs/portal/fr/index/news/publikationen.html?publicationID=3411)

Orchard, A. L., \& Solberg, K. B. (1999). Expectations of the stepmother's role. Journal of Divorce \& Remarriage, 31 , $107-123$.

Orleans, M., Palisi, B. J., \& Caddell, D. (1989). Marriage adjustment and satisfaction of stepfathers: Their feelings and perceptions of decision making and stepchildren relations. Family Relations, 38, 371-377.

Papernow, P. L. (1984). The stepfamily cycle: An experiential model of stepfamily development. Family Relations, 33 , $355-363$. 
Papernow, P. L. (2008). A clinician's view of "Stepfamily Architecture": Strategies for meeting the challenges. In J. Pryor (Ed.), The international handbook of stepfamilies: Policy and practice in legal, research, and clinical environments (pp. 423-454). New Jersey: John Wiley \& Sons.

Parent, C., Beaudry, M., Saint-Jacques, M. C., Turcotte, D., Robitaille, C., Boutin, M., \& Turbide, C. (2008). Les représentations sociales de l'engagement parental du beau-père en famille recomposée. Enfances, Familles, Générations, $154-171$.

Pink, J. E. T., \& Wampler, K. S. (1985). Problem areas in stepfamilies: Cohesion, adaptability, and the stepfather-adolescent relationship. Family Relations, 34, 327-335.

Pryor, J. (2008). The international handbook of stepfamilies: Policy and practice in legal, research, and clinical environments. New Jersey: John Wiley \& Sons.

Robertson, J. (2008). Stepfathers in families. In J. Pryor (Ed.), The international handbook of stepfamilies: Policy and practice in legal, research, and clinical environments. (pp. 125-150). New Jersey: John Wiley \& Sons.

Saint-Jacques, M. C. (1990). Familles recomposées : qu'avons-nous appris au fil des ans ? Service social, $39,7-37$.

Sauer, L., \& Fine, M. (1988). Parent-child relationships in stepfamilies. Journal of Family Psychology, 1, 434-451.

Schoppe-Sullivan, S. J., Mangelsdorf, S. C., Frosch, C. A., \& McHale, J. L. (2004). Associations between coparenting and marital behavior from infancy to the preschool years. Journal of Family Psychology, 18, 194-207.

Schrodt, P., \& Braithwaite, D. O. (2011). Coparental communication, relational satisfaction, and mental health in stepfamilies. Personal Relationships, 18, 352-369.

Smith, M. (2008). Resident mothers in stepfamilies. In J. Pryor (Ed.), The international handbook of stepfamilies: Policy and practice in legal, research, and clinical environments (pp. 151-174). New Jersey: John Wiley \& Sons.

Stewart, S. D. (1999). Disneyland dads, Disneyland moms? How nonresident parents spend time with absent children. Journal of Family Issues, 20, 539-556.

Théry, I., \& Dhavernas, M. J. (1993). La parenté aux frontières de l'amitié : statut et rôle du beau-parent dans les familles recomposées. In M. T. Meulders-Klein (Ed.), Les recompositions familiales aujourd'hui (pp. 159-187). Paris: Nathan.

Van Eeden-Moorefield, B., \& Pasley, K. (2008). A longitudinal examination of marital processes leading to instability in remarriages and stepfamilies. In J. Pryor (Ed.), The international handbook of stepfamilies: Policy and practice in legal, research, and clinical environments (pp. 231-249). New Jersey: John Wiley \& Sons.

Visher, E. B., \& Visher, J. S. (1993). Remarriage families and stepparenting. In F. Walsh (Ed.), Normal family processes (pp. 235-253). New York: Guilford Press.

Walsh, F. (1991). Promoting healthy functioning in divorced and remarried families. In A. S. Gurman, \& D. P. Kniskern (Eds.), Handbook of family therapy (2) (pp. 525-545). New York: Routledge.

White, L., \& Gilbreth, J. G. (2001). When children have two fathers: Effects of relationships with stepfathers and noncustodial fathers on adolescent outcomes. Journal of Marriage and Family, 63, 155-167.

White, L. K., \& Booth, A. (1985). The transition to parenthood and marital quality. Journal of Family Issues, 6, $435-449$.

Widmer, E. D., Favez, N., Aeby, G., De Carlo, I., \& Doan, M. T. (2012). Capital social et coparentage dans les familles recomposées et de première union. Sociograph 13. Genève: Université de Genève. 